\title{
Hybrid indium tin oxide-Au metamaterial as a multiband bi-functional light absorber in the visible and near-infrared ranges
}

\author{
Ataollah Kalantari Osgouei ${ }^{1,2, *} \mathbb{D}$, Hodjat Hajian ${ }^{2}$ (D), Andriy E Serebryannikov ${ }^{2,5}$ \\ and Ekmel Ozbay ${ }^{1,2,3,4}$ \\ ${ }^{1}$ Department of Physics, Bilkent University, Ankara 06800, Turkey \\ 2 NANOTAM- Nanotechnology Research Center, Bilkent University, Ankara 06800, Turkey \\ ${ }^{3}$ Department of Electrical and Electronics Engineering, Bilkent University, Ankara 06800, Turkey \\ ${ }^{4}$ UNAM- National Nanotechnology Research Center, Bilkent University, Ankara 06800, Turkey \\ ${ }^{5}$ Faculty of Physics, Adam Mickiewicz University, 61-614, Poznan, Poland \\ E-mail: akalantari@bilkent.edu.tr
}

Received 17 December 2020, revised 24 March 2021

Accepted for publication 7 April 2021

Published 23 April 2021

\begin{abstract}
Metamaterial nearly perfect light absorbers (MPAs) with dual-narrowband functionality-that absorb light in two narrowband adjacent wavelength regions-have attracted considerable attention due to their intriguing applications, such as sensing, photovoltaic, and thermal emission. Here, we propose a multi-band MPA with two narrowband absorption responses that are centered on the visible and near-infrared (NIR) wavelengths $(773 \mathrm{~nm}$ and $900 \mathrm{~nm}$, respectively) and a broadband absorptive characteristic in another window in the NIR region (ranging from $1530 \mathrm{~nm}$ to $2700 \mathrm{~nm}$ with a bandwidth of $1170 \mathrm{~nm}$ ). The MPA comprises a periodic array of self-aligned hybrid indium tin oxide (ITO)-Au split-ring-resonators that are separated from an optically thick bottom reflector with a $\mathrm{SiO}_{2}$ layer. Based on numerical calculations, which are accompanied with a semi-analytical examination, we find that the dual narrowband and broadband responses are attributed to the hybridization of the optical responses of gold as a plasmonic material with the ones of ITO. Note that ITO acts as a low-loss dielectric in the visible range and a lossy plasmonic material in the NIR region. Moreover, due to the applied symmetry in the unit cell of the metamaterial, the proposed MPA represents polarization insensitive and omnidirectional absorptive features. The proposed metastructure can find potential applications in selective thermophotovoltaic devices, thermal emitters, and sensors.
\end{abstract}

Keywords: hybrid metamaterials, light absorbers, visible, near infrared

(Some figures may appear in colour only in the online journal)

\section{Introduction}

Metamaterials are engineered structures that exhibit extraordinary electromagnetic properties not available in nature $[1,2]$. They can be employed to achieve various

\footnotetext{
Author to whom any correspondence should be addressed.
}

intriguing phenomena such as invisible cloaking [3], asymmetric light transmission [4], lasing [5], and negative refraction [6]. Light absorption is another extraordinary potential capability of these artificial structures [7]. Metamaterials with nearly perfect absorption features are generally called metamaterial nearly perfect light absorbers (MPAs). In 2008, Landy et al proposed a narrowband MPA based on metallic split ring resonator arrays [8]. Thereafter, considerable 
attention has been paid in research focusing on MPAs by many research groups during the past years. Depending on the geometry and the material type being employed in the MPA structures, their absorptive responses can be categorized as narrowband or broadband. The former category may have applications in sensing [9], imaging [10], and color filtering [11] while the latter category can be functionalized in photodetectors [12], solar cells [13], radiative coolers [14], and photochemical devices [15]. Such electromagnetic nearly perfect light absorbers have been reported in various frequency ranges that cover the visible [16, 17], near-infrared (NIR) [18, 19], mid-infrared (MIR) [20, 21], far-infrared [22, 23], terahertz [24, 25], and microwave regions [26-28]. In order to physically realize MPAs, as a strategy, reflectance can be suppressed by matching the effective impedance of the metamaterials with the surrounding medium. At the same time, transmission may be eliminated by employing an optically thick metal layer acting as a mirror [29, 30]. Therefore, one of the widely used approaches to obtain nearly perfect light absorption is to employ a metal-insulator-metal (MIM) configuration. Based on this structure, narrowband perfect absorption can be obtained for which the top layer of the MIM is either unpatterned [31] or patterned [32]. On the other hand, it is also possible to obtain broadband perfect absorption by employing either lossy materials like Ti [33] as a top patterned layer or using multidimensional objects in the top layer of each unit cell of the metamaterial $[34,35]$. In addition to the aforementioned structures that exhibit either narrowband or broadband responses, the MPAs with dual-narrowband functionality, which are absorptive in two different narrow wavelength regions and almost reflective in other bands have been widely studied by employing a single type of material on the top layer of the MIM structure [36-38]. Such designs are found to have practical applications in selective photonic structures, sensors and optoelectronic devices. The idea behind those designs is to employ meta-atoms based on a single type plasmonic material (e.g. Au) with different geometrical shapes. On the other hand, as a different approach, by hybridizing graphene as a plasmonic material and hexagonal boron nitride as a phononic material, MIR MPAs with multi-narrowband resonant responses have been obtained [39, 40]; i.e. employing diverse functional materials to achieve responses with different functionalities. However, to our knowledge, a hybrid MPA with meta-atoms composed of different plasmonic layers that represents narrowband responses in the visible and broadband feature in the NIR has not been reported.

In this paper, we propose a hybrid nearly perfect light absorber that is capable of functioning as a narrowband light absorber at $773 \mathrm{~nm}(\mathrm{FWHM} \sim 60 \mathrm{~nm})$ in the visible and at $900 \mathrm{~nm}(\mathrm{FWHM} \sim 49 \mathrm{~nm})$ in the NIR range (see figure 5(b)), where FWHM stands for full width at half maximum. Moreover, the proposed metamaterial can operate as a broadband MPA within $1530 \mathrm{~nm}$ to $2700 \mathrm{~nm}$ in the NIR region; i.e. a bi-functional response is observed by the MPA. The top patterned layer of this novel metastructure is composed of array of self-aligned hybrid indium tin oxide (ITO)-Au split ring resonators (SRRs), that are separated from an optically thick bottom reflector with a $\mathrm{SiO}_{2}$ layer. We first numerically evaluate the primarily obtained result by investigating how ITO and $\mathrm{Au}$ incorporate in the total absorption response of the metamaterial. Moreover, replacing an effective top layer with the hybrid array and employing transfer matrix method (TMM), the numerical results are also verified. As complementary investigations, the mode profiles at some frequencies inside the narrowband and broadband regions also confirm that Fabry-Perot (FP) and propagating surface plasmon (PSP) resonances are responsible for the bi-functional operation of the metastructure. Finally, by increasing the in-plane symmetry of the metamaterial, the polarization-independent and omnidirectional response of the device for transverse electric (TE) and transverse magnetic (TM) oblique incidences are also verified. Despite the considerable amount of research that have been done in the field of metamaterial light absorbers, we believe that this study can be of interest to readers and find potential applications in sensing, selective thermophotovoltaics and thermal emission.

\section{Theoretical background}

In order to get more insight into the physical mechanism of the absorption responses of the designed metamaterial, we employ the effective medium theory (EMT) together with TMM for a semi-analytic investigation of the numerically obtained absorption. In this approach, an effective homogeneous layer is replaced with the top patterned layer of the MPA that is composed of an array of self-aligned hybrid ITO-Au SRRs.

The schematic of the hybrid ITO-Au based metamaterial is schematically illustrated in figure 1(a). A unit cell of the MPA is also depicted in figure 1(c). As mentioned above, the metamaterial is composed of array of self-aligned hybrid ITO$\mathrm{Au}$ SRRs that is separated by an optically thick gold reflector with an $\mathrm{SiO}_{2}$ layer with thickness of $t_{\mathrm{SiO}_{2}}$. The ITO and Au layers thicknesses in each self-aligned SRR are also introduced as $t_{\mathrm{ITO}}$ and $t_{\mathrm{Au}}$. In the following we consider $t_{\mathrm{eff}}=t_{\mathrm{ITO}}+t_{\mathrm{Au}}$. The chosen values of the remained geometrical parameters of the unit cell of the metamaterial are also highlighted in panel (c) of figure 1. In order to semi-analytically analyze absorption of the metamaterial, we employ the TMM approach on the layered medium that schematically shown in figure 1(b). In this layered structure, an effective isotropic and homogeneous medium is replaced with the top patterned ITO-Au layer, as seen from figure 1. Here, absorption is calculated as $A=1-R$ where $A$ and $R$ denote light absorption and reflection, respectively. It should be noted that since an optically thick Au layer is chosen as the bottom reflector in the calculations, transmission is zero $(T=0)$. Considering the $\mathrm{z}$-component of the electric field as

$$
E_{z}(x)=\left\{\begin{array}{l}
A_{1} e^{-i k_{1}\left(z-t_{\mathrm{eff}}\right)}+B_{1} e^{i k_{1}\left(z-t_{\mathrm{eff}}\right)}, z>t_{\mathrm{eff}} \\
A_{2} e^{-i k_{2} z}+B_{2} e^{i k_{2} z}, 0<z<t_{\mathrm{eff}} \\
A_{3} e^{-i k_{3}\left(z+t_{\mathrm{eff}}\right)}+B_{3} e^{i k_{3}\left(z+t_{\mathrm{eff}}\right)},-t_{\mathrm{SiO}_{2}}<z<0 \\
A_{4} e^{-i k_{A u}\left(z+t_{\mathrm{eff}}+t_{\mathrm{Si}_{2}}\right)}, z<-t_{\mathrm{SiO}_{2}}
\end{array}\right.
$$




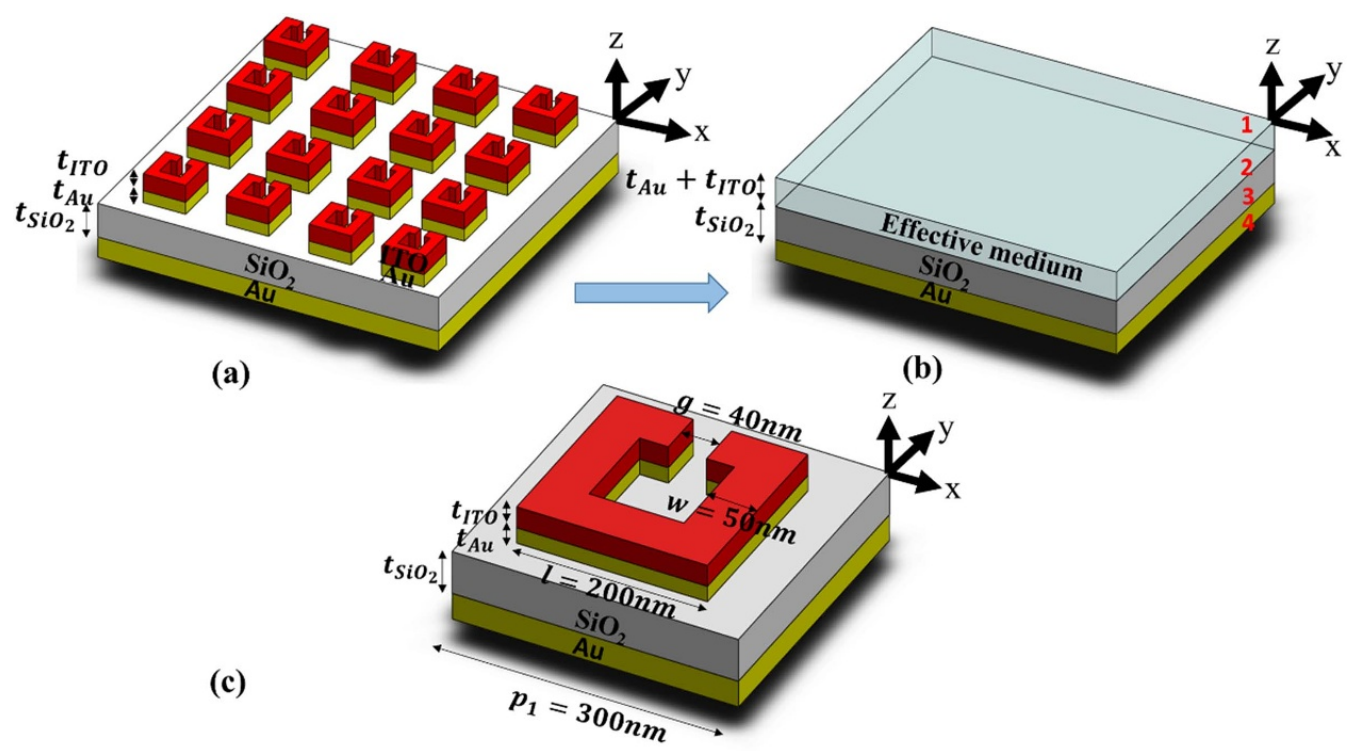

Figure 1. (a) Schematic of the self-aligned hybrid ITO-Au metamaterial nearly perfect light absorber. The unit cell of the metamaterial is depicted in panel (c). Panel (b) represents the optically equivalent model of the MPA shown in panel (a) in which the top effective medium layer is replaced by the self-aligned ITO-Au SRR array. The geometrical parameters and their corresponding are highlighted in panel (c). The values of the thicknesses of the layers are addressed in the text.

and imposing the boundary conditions at the interfaces [41], reflection of light can be obtained as $R=\left|M_{21} / M_{11}\right|^{2}$. Here, $k_{i}=\sqrt{\left(\frac{n_{i} \omega}{c}\right)^{2}-k_{x}^{2}}(i=1, \ldots, 4)$ (see figure 1(b) where the layers are labeled by numbers), $c$ is the speed of light, $n_{i}$ is the refractive index of each medium, and

$$
M=\left[\begin{array}{ll}
M_{11} & M_{12} \\
M_{21} & M_{22}
\end{array}\right]=D_{1}^{-1} D_{2} P_{2} D_{2}^{-1} D_{3} P_{3} D_{3}^{-1} D_{4}
$$

Here

$$
D_{i}=\left[\begin{array}{cc}
1 & 1 \\
n_{i} & -n_{i}
\end{array}\right]
$$

and the effect of light propagation through the effective medium $\left(n_{2}=n_{\text {eff }}\right)$ and the spacer layer $\left(n_{3}=n_{\mathrm{SiO}_{2}}\right)$ are taken as the following propagation matrices into account

$$
\begin{gathered}
P_{2}=\left[\begin{array}{cc}
e^{i \varphi_{2}} & 0 \\
0 & e^{-i \varphi_{2}}
\end{array}\right], \\
P_{2}=\left[\begin{array}{cc}
e^{i \varphi_{3}} & 0 \\
0 & e^{-i \varphi_{3}}
\end{array}\right]
\end{gathered}
$$

where $\varphi_{i}=n_{i} t_{i} \omega / c$ for the case of normal incidence. It is practical to replace a collection of periodically arranged scattering objects with subwavelength dimensions by a homogeneous medium. This homogenization approach, which is also called EMT, can be valid as far as the applied fields are static or have spatial variations on a scale larger than the scale of the inhomogeneous medium [42-44]. Consequently, the effective refractive index of the effective medium in figure 1(b) can be retrieved using $S_{11}$ and $S_{21}$ scattering elements of normally incident reflected and transmitted light. Due to the presence of the thick metallic substrate $S_{21}=0$ and $S_{11}$ will be calculated numerically [45].

\section{Results and discussions}

In this section, we investigate the absorption response of the MPA shown in figure 1 in a broad range of wavelengths covering the visible and NIR regions $(400 \mathrm{~nm}-3500 \mathrm{~nm})$. Note that, considering the previously reported practical parameters of the SRR metamaterials [46-48], we performed geometrical parameter optimization simulations to obtain the following feasible parameter. Based on these parameters and employing nanofabrications techniques $[49,50]$, the suggested designs in this study can be experimentally verified. Therefore, the geometrical parameters shown in figure 1(c) are taken as $w=50 \mathrm{~nm}, g=40 \mathrm{~nm}, l=200 \mathrm{~nm}, t_{\mathrm{Au}}=60 \mathrm{~nm}$, $t_{\mathrm{ITO}}=80 \mathrm{~nm}, t_{\mathrm{SiO}_{2}}=160 \mathrm{~nm}$, and $p_{1}=300 \mathrm{~nm}$, unless stated otherwise. The thickness of the optically thick bottom reflector is also chosen as $500 \mathrm{~nm}$ in the simulations. Refractive index of $\mathrm{SiO}_{2}$ and $\mathrm{Au}$ are also selected based on experimental values [51, 52]. Moreover, following the experimental data [53], ITO has been taken into account using the Drude dispersion relation $\varepsilon(\omega)=\varepsilon_{\infty}-\omega_{\mathrm{p}}^{2} /\left[\omega\left(\omega+i \gamma_{\mathrm{p}}\right)\right]$, with the background permittivity of $\varepsilon_{\infty}=3.91$, the plasma frequency of $\omega_{\mathrm{p}}=2.65 \times 10^{15} \mathrm{rad} \mathrm{s}^{-1}$, and collision frequency of $\gamma_{p}=2.05 \times 10^{14} \mathrm{rad} \mathrm{s}^{-1}$. It is noteworthy that, for the finitedifference time-domain simulations, the considered unit cell is excited with a plane wave, and periodic boundary condition (perfectly matched layer) is applied in the $X-Y$ boundaries ( $Z$ boundaries).

The simulated absorption of the MPA exhibits a narrowband perfect resonant peak at $767 \mathrm{~nm}$ with a high quality factor $(\sim 13$, FWHM $\sim 59 \mathrm{~nm})$ and a broadband perfect absorption spanning from $1790 \mathrm{~nm}$ to $3000 \mathrm{~nm}$, as shown in the solidblue curve in figure 2(a). More investigations prove that (results are not shown here) if the hybrid ITO-Au SRR array is replaced with an array of periodic hybrid ITO-Au disks with 
(a)

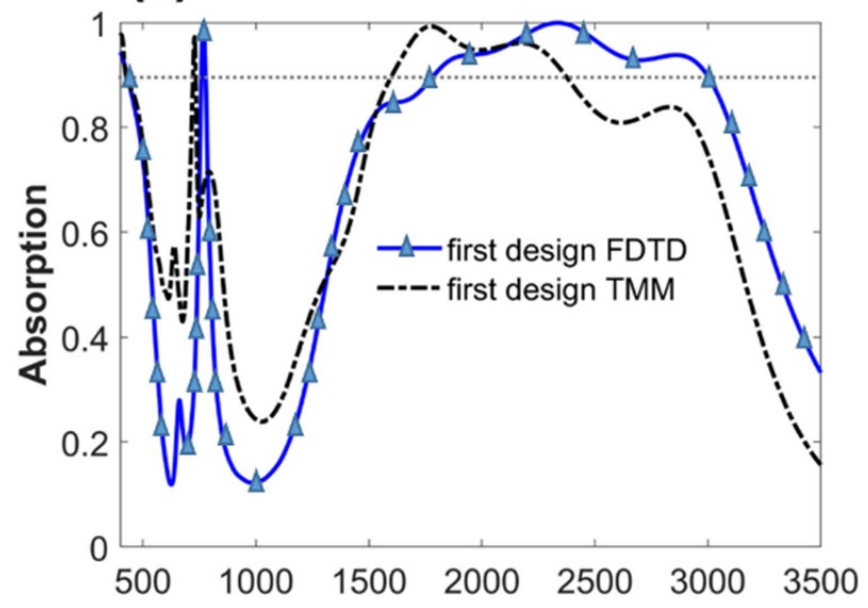

(b)

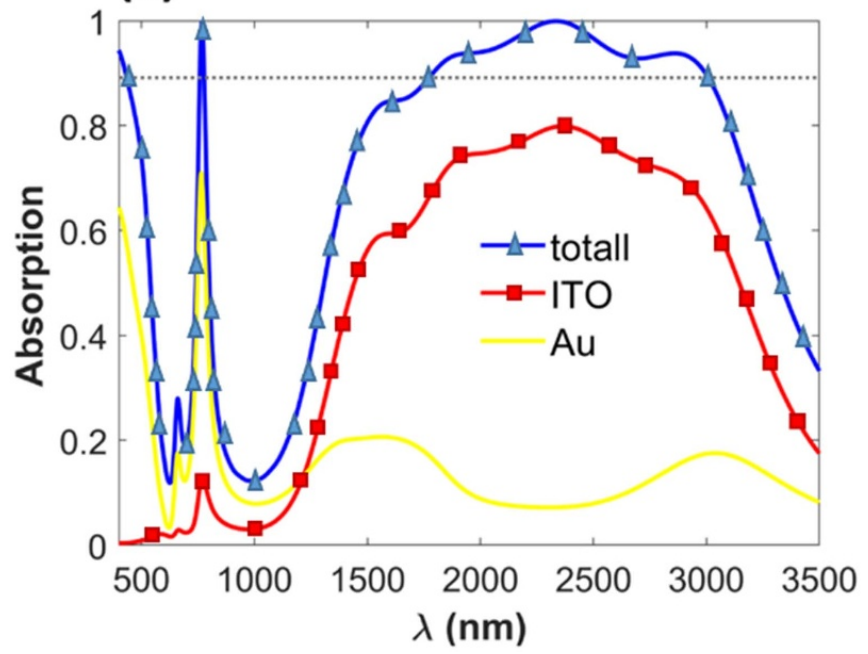

Figure 2. (a) Simulated normal incidence absorption spectra of the actual MPA (solid-blue) and the corresponding semi-analytical result based on the EMT and TMM. (b) Contribution of the ITO SRR and Au SRR in the total absorption of the self-aligned hybrid ITO-Au SRR. The results are obtained for normal incidence of light.

$r=100 \mathrm{~nm}$, two narrow absorption peaks in the visible and near infrared regions will be obtained. In other words, due to its geometrical characteristic, the metamaterial composed of the hybrid SRR array can more efficiently harvest the incident light in the NIR region to achieve broadband absorption response, as compared to an array of disks. Moreover, by further investigations of the results we understood that for the case of $g=40 \mathrm{~nm}$, the NIR absorption response of the metamaterial is broaden than $g=80 \mathrm{~nm}$ and $g=100 \mathrm{~nm}$ cases. However, by removing the gap (i.e. $g=100 \mathrm{~nm}$ ) it is still possible to obtain a broadband NIR absorption.

To understand the physical mechanism of the nearly perfect absorption responses of the structure, we employ the EMT together with the TMM on the layered structure depicted in figure 1(b). As mentioned earlier, in the semi-analytical approach, the effective refractive index $n_{\text {eff }}$ of the hybrid ITOAu SRR array with the effective thickness of $t_{\text {eff }}$ are taken into account. The result is illustrated as the dashed-dotted black curve in figure 2(a) which fairly matches with the perfect narrow-band absorption wavelength. This agreement indicates the verification of the FP resonance by the applied approach. There is also an acceptable agreement between the semianalytical and numerical result in the broadband region confirming that replacing a homogenized medium with the top ITO-Au patterned layer is justified for this case. However, the observed discrepancies between the semi-analytical and the numerical results at $\lambda>2250 \mathrm{~nm}$ notifies that, at larger wavelengths, the PSP resonances are mostly responsible for the absorption response of the MPA that are less captured by the homogenization approach. This point is clarified by examining the mode profiles in figure 3.

In order to achieve more understanding about the absorption mechanism of the hybrid ITO-Au SRR array, contribution of the ITO and Au SRRs in the total absorption of the hybrid MPA is investigated in figure 2(b). The solid-yellow curve in this figure clearly highlights the role of gold, as a noble metal with moderate losses, in the appearance of the narrowband absorption resonance of the MPA at $\lambda=767 \mathrm{~nm}$. However, ITO has a minimum contribution in appearance of that resonance since it behaves as a low-loss dielectric material at the corresponding wavelength. On the other hand, once the solid-red curve is compared to the total absorption of the MPA in figure 2(b), it is understood that ITO, that can act as a lossy plasmonic material at $\lambda>1420 \mathrm{~nm}$, is responsible for the broadband response of the metamaterial absorber. It is observed that the hybridization of the ITO and Au SRR array in the final MPA eventually provides a nearly perfect narrowband resonant peak and a broadband absorption response in the NIR region.

Investigation of the mode profiles at the resonance wavelengths is also an effective tool to reveal the physical mechanisms behind the absorption characteristics of the proposed MPA. With this aim, electric and magnetic field profiles at the narrowband resonant absorption wavelength $(\lambda=767 \mathrm{~nm})$ and three resonant wavelengths in the broadband region, namely $\lambda=1780 \mathrm{~nm}, 2,314 \mathrm{~nm}$, and 2,800 nm, are shown in figure 3 .

Top-view of $|E|$, side-view of $|E|$, and side-view of $|H|$ at the resonant wavelengths are represented in panels $\left(\mathrm{a}_{\mathrm{i}}\right),\left(\mathrm{b}_{\mathrm{i}}\right)$ and $\left(c_{i}\right)$ of figure 3 , respectively. The top- and side-view mode profiles of $|E|$ at $767 \mathrm{~nm}$ (panels $\left(\mathrm{a}_{1}\right)$ and $\left(\mathrm{b}_{1}\right)$ ) shows the electric field is mostly localized at the gap and between the edges of the SRR arms while from panel $\left(c_{1}\right)$ it is seen that the magnetic field is mostly localized at the edges of the Au SRR array. This observation conveys that, at this wavelength, FP resonance is responsible for the narrowband perfect absorption. Panels $\left(\mathrm{a}_{2}\right)$ and $\left(b_{2}\right)$ of figure 3 represent that the electric field at $1780 \mathrm{~nm}$ is localized between the arms of the ITO SRR while the magnetic field is captured in the gold region with weak interaction with the bottom reflector. This observation verifies that due to the localization of the electric field in the ITO SRR, which is a lossy material at that wavelength, a low-Q resonance is responsible for the absorption. The same point can be observed from the top- and side-view of $|E|$ mode profiles at $2314 \mathrm{~nm}$ and $2800 \mathrm{~nm}$. However, from the $|H|$ mode profiles in panels $\left(c_{3}\right)$ and $\left(c_{4}\right)$, it is observed that there are strong interactions between the magnetic fields and the bottom reflector at those 

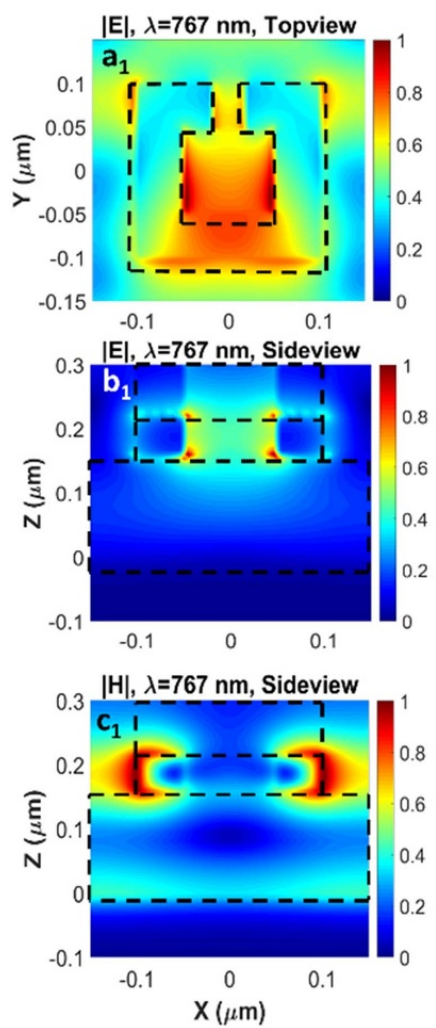
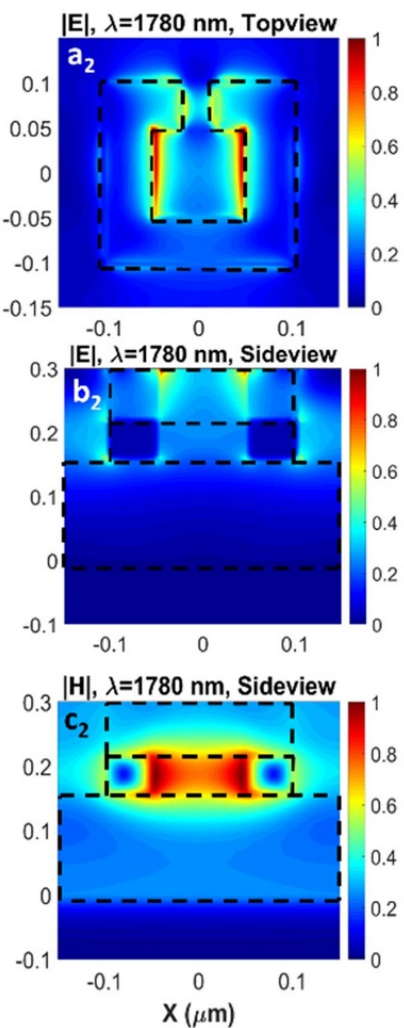
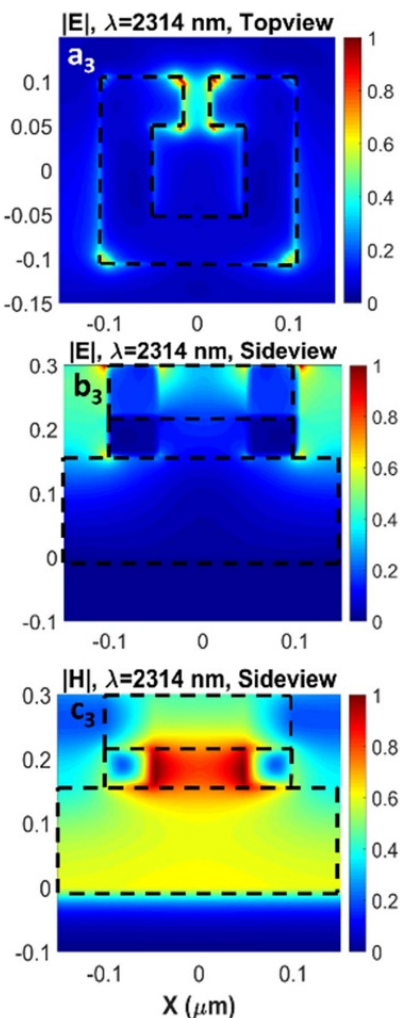
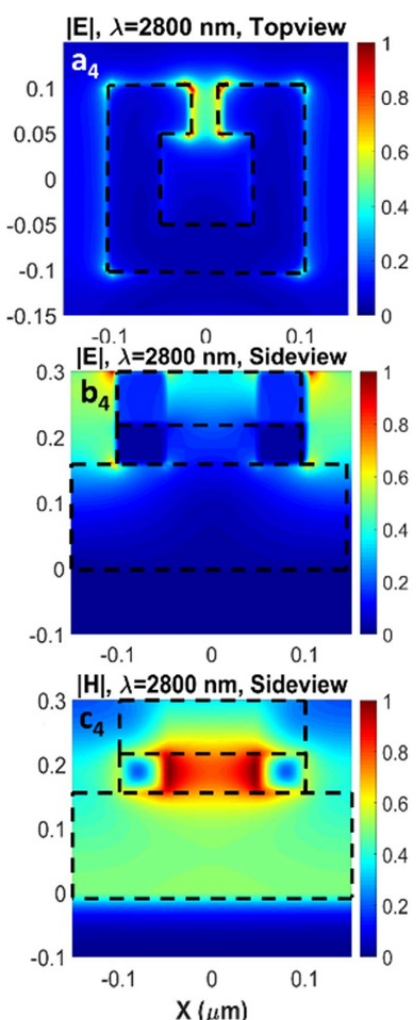

Figure 3. Panels $\left(\mathrm{a}_{\mathrm{i}}\right),\left(\mathrm{b}_{\mathrm{i}}\right)$, and $\left(\mathrm{c}_{\mathrm{i}}\right)(i=1,2,3)$, respectively, illustrate normalized values of the top-view $|E|$ mode profiles, side-view of $|E|$ and side view of $|H|$ at the narrowband resonant peak $(767 \mathrm{~nm})$ and the three different absorption resonances in the broadband region, namely $1780 \mathrm{~nm}, 2314 \mathrm{~nm}$, and $2800 \mathrm{~nm}$. The dashed rectangles in panels $\left(\mathrm{b}_{\mathrm{i}}\right)$ and $\left(\mathrm{c}_{\mathrm{i}}\right)$ highlight the position of the ITO, Au and SiO $\mathrm{I}_{2}$ layers.

wavelengths. In fact, the magnetic fields in these panels are not only confined in the region beneath each SRR, but they are also enhanced in the entire spacer region. This behavior indicates the role of PSP resonances in the absorptive responses of the MPA at larger wavelengths. In fact, a combination of lowQ resonances with the PSP ones at different wavelengths in the broadband region causes the broadband light absorption by the MPA.

In the next step, we examine how changes in $t_{\mathrm{SiO}_{2}}, t_{\mathrm{Au}}$ and $t_{\text {ITO }}$ can affect light absorption of the metamaterial.

Panels (a), (b), and (c) of figure 4 shows dependence of the absorbed light by MPA on the changes in $t_{\mathrm{SiO}_{2}}, t_{\mathrm{Au}}$ and $t_{\mathrm{ITO}}$, respectively, while keeping all the other parameters the same as the aforementioned optimized/feasible values. One can observe in figure 4(a) that deviation from the optimized value (i.e. $t_{\mathrm{SiO}_{2}}=160 \mathrm{~nm}$ ) of the spacer layer leads to either losing the NIR broadband response (for $t_{\mathrm{SiO}_{2}}<160 \mathrm{~nm}$ ) or depriving the narrowband absorption in the visible region (for $t_{\mathrm{SiO}_{2}}>160 \mathrm{~nm}$ ). As mentioned hereinabove, the Au and ITO self-aligned SRRs are responsible for the narrowband and broadband absorptive response of the MPA. In agreement with that point, figure 4(b) shows that decreasing $t_{\mathrm{Au}}$ red-shifts the narrowband resonance (compared to the optimized case of $t_{\mathrm{Au}}=60 \mathrm{~nm}$ ), broadens it and leads to a slight loss in the broadband response. On the other hand, choosing thicker values of the gold thickness has a negligible effect on the broadband response while it slightly blue shifts the narrowband resonance. Moreover, the decreasing thickness of ITO from the optimized value (i.e. $t_{\mathrm{ITO}}=80 \mathrm{~nm}$ ) reduces the bandwidth while it has less effect on the narrowband resonance, see figure 4(c). As a result, considering an absorption above $90 \%$ for the nearly perfect efficiency of our structure, the case of $160 \mathrm{~nm} / 60 \mathrm{~nm} / 80 \mathrm{~nm}$ for $t_{\mathrm{SiO}_{2}} / t_{\mathrm{Au}} / t_{\text {ITO }}$ gives the most promising optimum absorption with a bandwidth of $1210 \mathrm{~nm}$ (from $1790 \mathrm{~nm}$ to $3000 \mathrm{~nm}$ ) and a nearly perfect absorption at the wavelength of $767 \mathrm{~nm}$.

The proposed design (shown in figure 1(c)) lacks polarization insensitivity that is the key characteristic for absorption features of the practical MPAs. Therefore, to resolve this deficiency, we improve the aforementioned design to a polarization independent one by enhancing the structural symmetry.

A schematic of the symmetric unit cell composed of four self-aligned ITO-Au hybrid SRRs that are mutually rotated by $45^{\circ}$ is depicted in figure 5(a). We keep all the geometrical parameters of each SRR in the symmetric unit cell as the optimized values of the nonsymmetrical design. The only modified parameter is the periodicity; i.e. $p_{2}=600 \mathrm{~nm}$ in the calculations. Normal incidence absorption of the symmetric design is represented as the dashed-red curve in figure 5(b). Moreover, for the sake of comparison, the simulated absorption of the MPA of figure 1 is also shown as the solid blue curve. It is observed from the dashed-red curve in figure 5(b) that a broadband nearly perfect absorption (ranging from $1530 \mathrm{~nm}$ to $2700 \mathrm{~nm}$ ) and two narrowband nearly perfect absorption peaks at $773 \mathrm{~nm}(\mathrm{FWHM} \sim 60 \mathrm{~nm})$ and $900 \mathrm{~nm}(\mathrm{FWHM} \sim 49 \mathrm{~nm})$ are obtained. Therefore, by employing the symmetric MPA, 


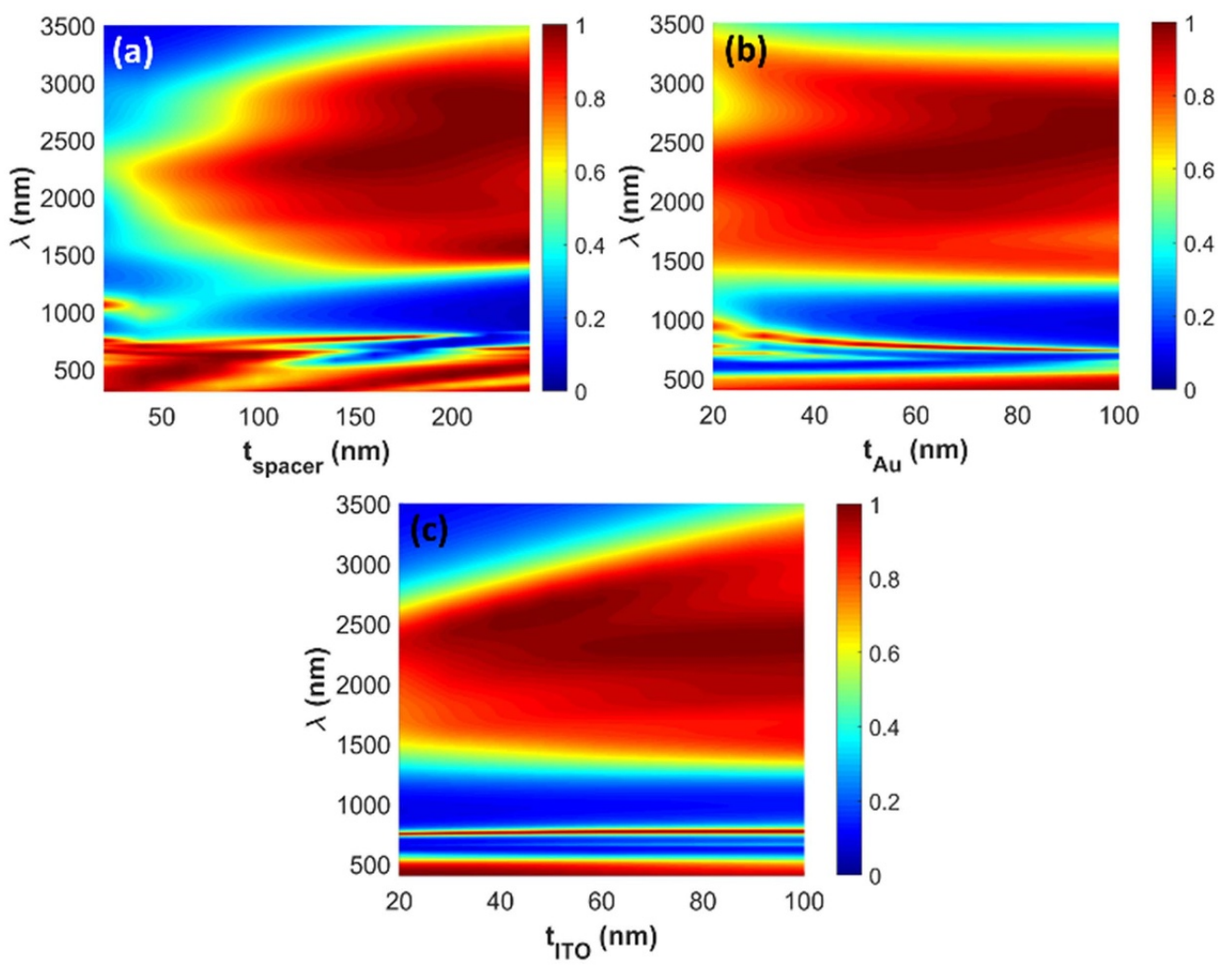

Figure 4. Absorption spectra of the MPA, that is schematically shown in figure 1, with respect to the thickness of spacer (a), thickness of the Au SRR (b) and thickness of the ITO SRR (c). Note that in panel (a) $t_{\mathrm{Au}}=60 \mathrm{~nm}$ and $t_{\mathrm{ITO}}=80 \mathrm{~nm}$, in panel (b) $t_{\mathrm{SiO}_{2}}=160 \mathrm{~nm}$ and $t_{\mathrm{ITO}}=80 \mathrm{~nm}$, and in panel (c) $t_{\mathrm{SiO}_{2}}=160 \mathrm{~nm}$ and $t_{\mathrm{Au}}=60 \mathrm{~nm}$.
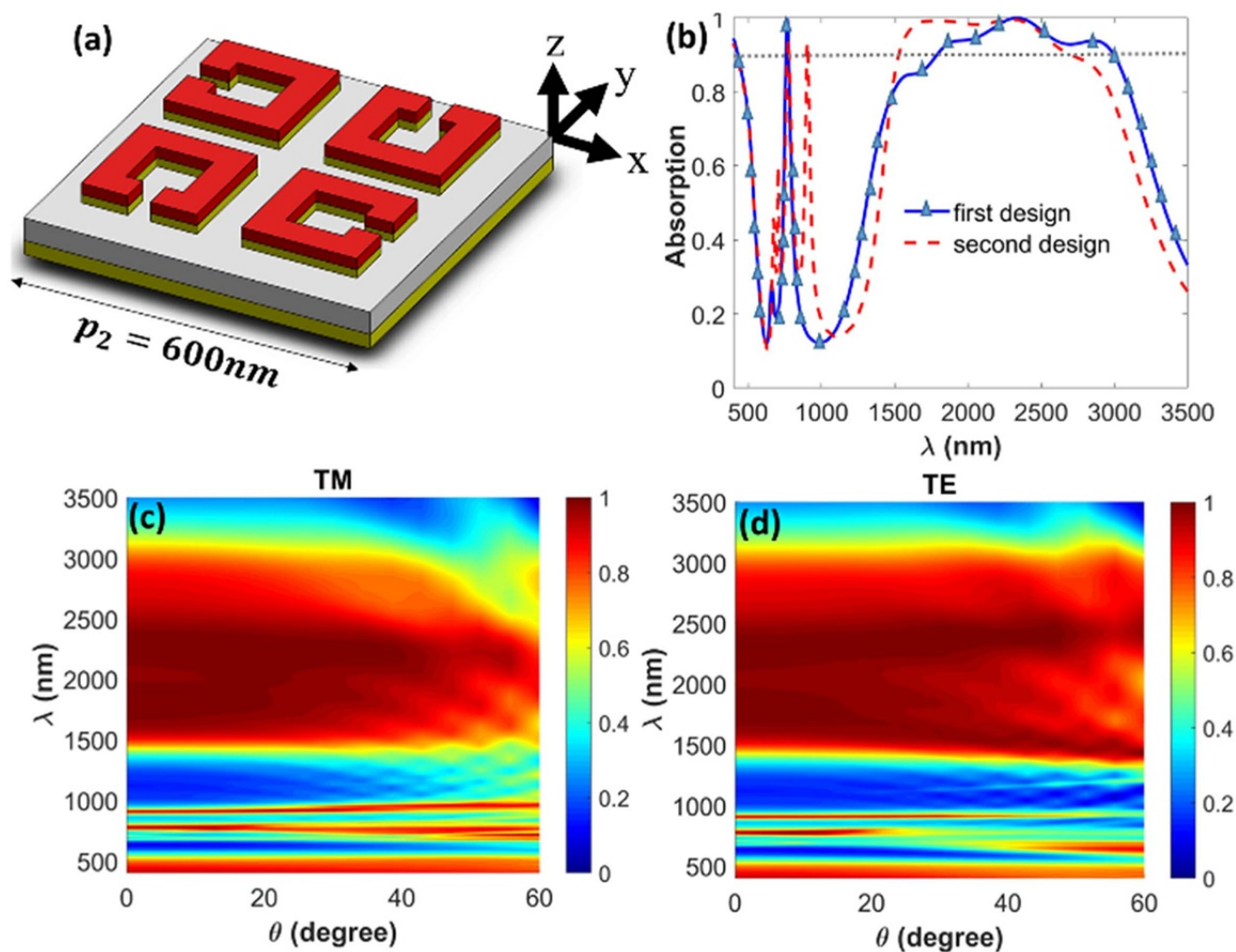

Figure 5. (a) Schematic of a unit cell of the symmetric MPA. This unit cell is composed of four self-aligned ITO-Au SRRs that are mutually rotated by $45^{\circ}$. Numerically calculated absorption of the symmetric metamaterial for normal incidence is shown by the dashed-red curve in panel (b). For the sake of comparison, the absorption of the nonsymmetric metamaterial of figure 1(c) is also illustrated again as the solid-blue curve. Figures (c) and (d), respectively, represent the dependence of the absorption performance of the symmetric structure on the incident angle for TM and TE polarizations. 

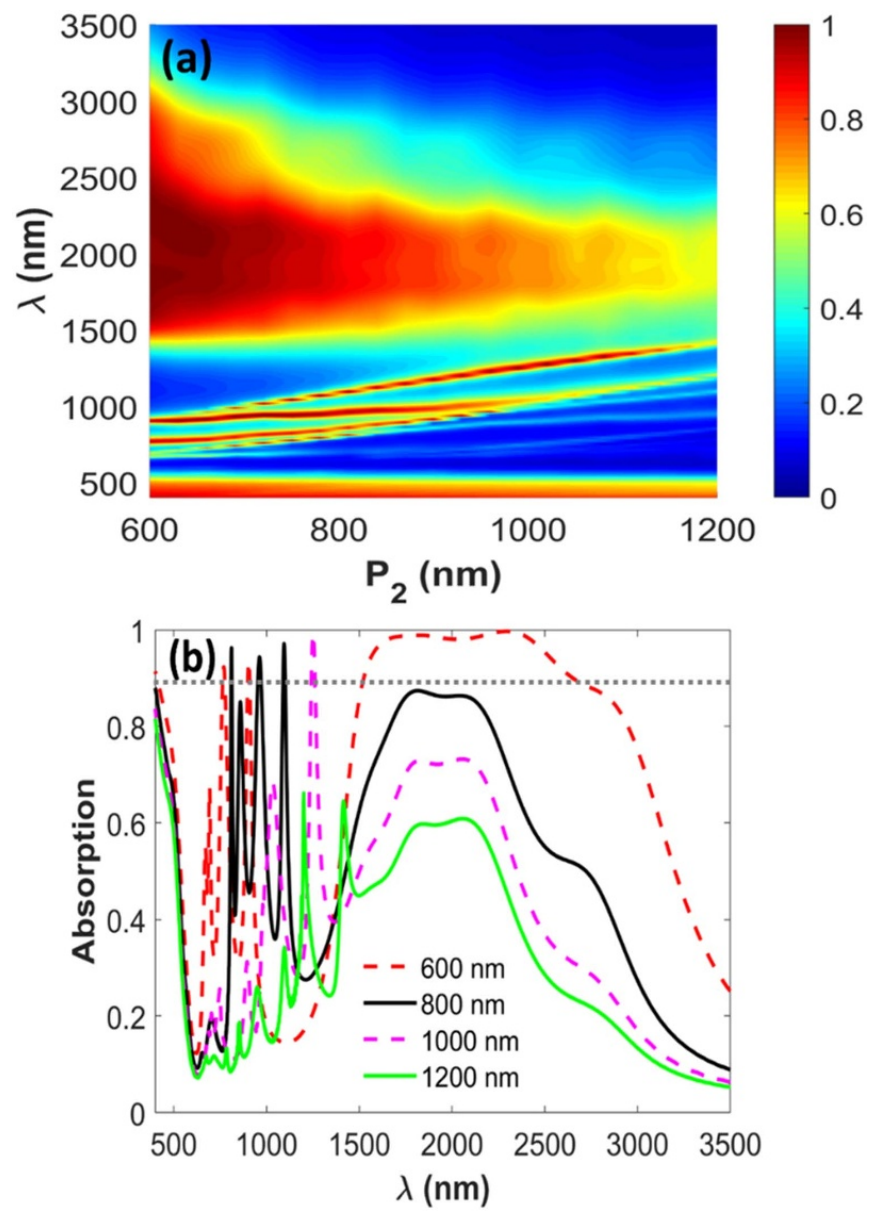

Figure 6. (a) Spectral absorption of the symmetric MPA as the function periodicity of the metamaterial, $p_{2}$. In agreement with panel (a), the absorption spectra for four different values of $p_{2}$ are shown in panel (b). Note that the geometrical parameters are chosen as the optimized ones mentioned in the text. The results are provided for normal incidence.

in addition to achieving a broadband absorption in the NIR and a narrowband absorption in the visible, one additional narrow band absorption peak is also obtained. The additional narrow band response, in fact, improves the functionality of the symmetric structure upon the nonsymmetrical one. More importantly, it is observed in figures 5(c) and (d) that the nearly perfect and broadband absorption of the symmetric MPA is preserved up to 50 degrees of incident angle for both TM and TE polarizations. The strength of the narrow band absorption resonances of the MPA are also kept unaffected for TM polarization while they are sensitive for angles of incidence more than $20^{\circ}$ for TE polarization. Specifically, for TE polarization, there is only a single nearly perfect absorption peak at $\theta=30^{\circ}$ around $900 \mathrm{~nm}$ while at $\theta=50^{\circ}$ only one nearly perfect resonance is observed around $773 \mathrm{~nm}$.

The last point that should be addressed here is the physical mechanism behind the appearance of the additional resonance at $900 \mathrm{~nm}$ in the MPA with the symmetric unit cell. This point is discussed in figure 6.

In figure 6 the impact of changes in the periodicity $\left(600 \mathrm{~nm}<p_{2}<1200 \mathrm{~nm}\right)$ on the overall normal incidence absorption characteristics of the MPA is investigated. Here, all other geometrical parameters are kept as the aforementioned optimized values. For the sake of feasibility, the minimum value of the periodicity is chosen as $600 \mathrm{~nm}$. As already explained, once the periodicity is chosen as this value, a broadband response in the NIR region together with two narrowband absorption peaks - in the visible and NIR regions - are obtained. As figure 6(b) clearly shows, by increasing the periodicity, the structure loses its broadband absorptive feature. As an example, once the periodicity is taken as $1200 \mathrm{~nm}$, the broadband feature is totally lost. On the other hand, the dependence of the narrowband response of the metamaterial to the increase in $p_{2}$ is somehow different than the broadband response. By increasing $p_{2}$ from $600 \mathrm{~nm}$ to $800 \mathrm{~nm}$, the narrowband resonances are red-shifted and the three narrow band resonances appear, see figure 6(b). In fact, the physical mechanism behind the appearance of additional narrowband resonances is the mutual coupling effect between the SRRs in each symmetric unit cell and the adjacent unit cells. However, at $p_{2}=800 \mathrm{~nm}$ the broadband characteristic is considerably lost. This point is also evident once the red-dashed curve is compared to the solid-black one in figure 6(b). Therefore, as far as we are interested in keeping both broadband and narrowband responses as efficient as possible, $p_{2}=600 \mathrm{~nm}$ is the optimized choice. It is noteworthy that according to the Kirchhoff's law of thermal radiation, the spectral emissivity $(E)$ of a structure is equal to its spectral absorptivity; i.e. $A(\lambda)=E(\lambda)$. Consequently, the absorption features of our proposed device can be employed for the realization of thermal emission characteristics [54].

\section{Conclusions}

In summary, in this study, we have proposed a novel hybrid metastructure that functions as a narrowband MPA at two wavelength is the visible $(733 \mathrm{~nm})$ and NIR $(900 \mathrm{~nm})$ ranges, and operates as a broadband absorber in a wide range of wavelengths in another window in the NIR region (from $1530 \mathrm{~nm}$ to $2700 \mathrm{~nm}$ with the bandwidth of $1170 \mathrm{~nm}$ ). The metamaterial is composed of an array of self-aligned hybrid ITO-Au SRRs that are separated from an optically thick bottom reflector with a $\mathrm{SiO}_{2}$ spacer layer. ITO behaves as a low-loss dielectric material in the visible range and a lossy plasmonic material at wavelengths larger than $1420 \mathrm{~nm}$. Consequently, it is found out that the narrowband response of the metamaterial is originated from the low-loss plasmonic response of $\mathrm{Au}$ SRRs and the broadband absorptive characteristic is achieved owing to the lossy plasmonic feature of ITO. As mentioned earlier, since feasible parameters have been considered in optimizing the suggested design, it is possible to experimentally verify the numerical results reported in this study. More investigations revealed that the designed MPA represents polarization-independent and omnidirectional absorption characteristics for oblique incidences up to $50^{\circ}$. The proposal MPA can find potential applications in sensors, thermophotovoltaic devices and thermal emitters. 


\section{Data availability statement}

No new data were created or analyzed in this study.

\section{Acknowledgments}

One of the authors (A.E.S.) received partial financial support by Narodowe Centrum Nauki, Grant No. $\sim 2015 / 17 / B / S T 3 / 00118$ and TUBITAK under Program 2221.

\section{ORCID iDs}

Ataollah Kalantari Osgouei (i) https://orcid.org/0000-00020971-7687

Hodjat Hajian (10 https://orcid.org/0000-0001-6564-6273

\section{References}

[1] Boardman A 2010 Pioneers in metamaterials: John Pendry and Victor Veselago J. Opt. 13020401

[2] Tretyakov S, Urbas A and Zheludev N 2017 The century of metamaterials J. Opt. 19080404

[3] Valentine J, Li J, Zentgraf T, Bartal G and Zhang X 2009 An optical cloak made of dielectrics Nat. Mater. 8 568-71

[4] Li Z, Mutlu M and Ozbay E 2013 Chiral metamaterials: from optical activity and negative refractive index to asymmetric transmission J. Opt. 15023001

[5] Fang A, Koschny T and Soukoulis C M 2010 Lasing in metamaterial nanostructures J. Opt. 12024013

[6] Hoffman A J, Alekseyev L, Howard S S, Franz K J, Wasserman D, Podolskiy V A, Narimanov E E, Sivco D L and Gmachl C 2007 Negative refraction in semiconductor metamaterials Nat. Mater. $6946-50$

[7] Watts C M, Liu X and Padilla W J 2012 Metamaterial electromagnetic wave absorbers Adv. Mater. 24 OP98-120

[8] Landy N I, Sajuyigbe S, Mock J J, Smith D R and Padilla W J 2008 Perfect metamaterial absorber Phys. Rev. Lett. 100

[9] Shi L, Shang J, Liu Z, Li Y, Fu G, Liu X, Pan P, Luo H and Liu G 2020 Ultra-narrow multi-band polarization-insensitive plasmonic perfect absorber for sensing Nanotechnology 31465501

[10] Landy N I, Bingham C M, Tyler T, Jokerst N, Smith D R and Padilla W J 2009 Design, theory, and measurement of a polarization-insensitive absorber for terahertz imaging Phys. Rev. B 79

[11] Yokogawa S, Burgos S P and Atwater H A 2012 Plasmonic color filters for CMOS image sensor applications Nano Lett. 12 4349-54

[12] Li W and Valentine J 2014 Metamaterial perfect absorber based hot electron photodetection Nano Lett. 14 3510-4

[13] Liu Y, Chen Y, Li J, Hung T-C and Li J 2012 Study of energy absorption on solar cell using metamaterials Sol. Energy 86 1586-99

[14] Liu T and Takahara J 2017 Ultrabroadband absorber based on single-sized embedded metal-dielectric-metal structures and application of radiative cooling Opt. Express 25

[15] Ghobadi T G U, Ghobadi A, Ozbay E and Karadas F 2018 Strategies for plasmonic hot-electron-driven photoelectrochemical water splitting ChemPhotoChem 2 161-82

[16] Lin C-H, Chern R L and Lin H-Y 2011 Polarization-independent broad-band nearly perfect absorbers in the visible regime Opt. Express 19415
[17] Ghobadi A, Hajian H, Gokbayrak M, Dereshgi S A, Toprak A, Butun B and Ozbay E 2017 Visible light nearly perfect absorber: an optimum unit cell arrangement for near absolute polarization insensitivity Opt. Express 2527624

[18] Osgouei A K, Hajian H, Khalichi B, Serebryannikov A E, Ghobadi A and Ozbay E 2021 Active tuning from narrowband to broadband absorbers using a sub-wavelength $\mathrm{VO}_{2}$ embedded layer Plasmonics $1-9$

[19] Zhu Y, Lan T, Liu P and Yang J 2019 Broadband near-infrared $\mathrm{TiO}_{2}$ dielectric metamaterial absorbers Appl. Opt. 587134

[20] Zhang K, Deng R, Song L and Zhang T 2019 Broadband near-infrared absorber based on all metallic metasurface Materials 123568

[21] Deng B, Guo Q, Li C, Wang H, Ling X, Farmer D B and Xia F 2016 Coupling-enhanced broadband mid-infrared light absorption in graphene plasmonic nanostructures ACS Nano 10 11172-8

[22] Lin Y Y, Cui Y X, Ding F, Fung K H, Ji T, Li D D and Hao Y Y 2017 Tungsten based anisotropic metamaterial as an ultra-broadband absorber Opt. Mater. Express 7 606-17

[23] Liu H, Luo K, Tang S, Peng D, Hu F and Tu L 2018 An ultra-wideband THz/IR metamaterial absorber based on doped silicon Materials 112590

[24] Li J, Li J, Yang Y, Li J, Zhang Y, Wu L and Yao J 2020 Metal-graphene hybrid active chiral metasurfaces for dynamic terahertz wavefront modulation and near field imaging Carbon 163 34-42

[25] Li H, Xu W, Cui Q, Wang Y and Yu J 2020 Theoretical design of a reconfigurable broadband integrated metamaterial terahertz device Opt. Express 28 40060-74

[26] Nguyen T T and Lim S 2018 Design of metamaterial absorber using eight-resistive-arm cell for simultaneous broadband and wide-incidence-angle absorption Sci. Rep. 8

[27] Zhuang H, Wang X, Wang J, Guo M, Tang D, Zhang B, Chen X, Chen P and Yang Y 2020 Broadband microwave metamaterial absorber based on magnetic periodic elements J. Phys. D: Appl. Phys. $\mathbf{5 3} 255502$

[28] Huang Y, Luo J, Pu M, Guo Y, Zhao Z, Ma X, Li X and Luo X 2019 Catenary electromagnetics for ultra-broadband lightweight absorbers and large-scale flat antennas $A d v$. Sci. 61801691

[29] Hajian H, Ghobadi A, Butun B and Ozbay E 2019 Active metamaterial nearly perfect light absorbers: a review [Invited] J. Opt. Soc. Am. B 36 F131

[30] Chen H-T 2012 Interference theory of metamaterial perfect absorbers Opt. Express 207165

[31] Li Z, Butun S and Aydin K 2015 Large-area, lithography-free super absorbers and color filters at visible frequencies using ultrathin metallic films ACS Photonics 2 183-8

[32] Yong Z, Zhang S, Gong C and He S 2016 Narrow band perfect absorber for maximum localized magnetic and electric field enhancement and sensing applications Sci. Rep. 6

[33] Ding F, Dai J, Chen Y, Zhu J, Jin Y and Bozhevolnyi S I 2016 Broadband near-infrared metamaterial absorbers utilizing highly lossy metals Sci. Rep. 6

[34] Nielsen M G, Pors A, Albrektsen O and Bozhevolnyi S I 2012 Efficient absorption of visible radiation by gap plasmon resonators Opt. Express 2013312

[35] Ghobadi A, Hajian H, Rashed A R, Butun B and Ozbay E 2018 Tuning the metal filling fraction in metal-insulator-metal ultra-broadband perfect absorbers to maximize the absorption bandwidth Photonics Res. 6168

[36] Tang Y, Bossard J A, Werner D H and Mayer T S 2008 Single-layer metallodielectric nanostructures as dual-band midinfrared filters Appl. Phys. Lett. 92263106

[37] Jiang Z H, Yun S, Toor F, Werner D H and Mayer T S 2011 Conformal dual-band near-perfectly absorbing mid-infrared metamaterial coating ACS Nano 5 4641-7 
[38] Zhang B, Zhao Y, Hao Q, Kiraly B, Khoo I-C, Chen S and Huang T J 2011 Polarization-independent dual-band infrared perfect absorber based on a metal-dielectric-metal elliptical nanodisk array Opt. Express 1915221

[39] Hajian H, Ghobadi A, Butun B and Ozbay E 2018 Tunable, omnidirectional, and nearly perfect resonant absorptions by a graphene-hBN-based hole array metamaterial $O p t$. Express 2616940

[40] Li H and Yu J 2020 Bifunctional terahertz absorber with a tunable and switchable property between broadband and dual-band Opt. Express 28 25225-37

[41] Yeh P 2005 Optical Waves in Layered Media (Hoboken, NJ: Wiley-Interscience)

[42] Smith D R, Vier D C, Koschny T and Soukoulis C M 2005 Electromagnetic parameter retrieval from inhomogeneous metamaterials Phys. Rev. E 71

[43] Kriegler C E, Rill M S, Linden S and Wegener M 2010 Bianisotropic photonic metamaterials IEEE J. Sel. Top. Quantum Electron. 16 367-75

[44] Smith D R 2010 Analytic expressions for the constitutive parameters of magnetoelectric metamaterials Phys. Rev. E 81

[45] Lumerical Inc. (available at: www.lumerical.com/products/)

[46] Dicken M J, Aydin K, Pryce I M, Sweatlock L A, Boyd E M, Walavalkar S, Ma J and Atwater H A 2009 Frequency tunable near-infrared metamaterials based on $\mathrm{VO}_{2}$ phase transition Opt. Express 1718330
[47] Cakmakyapan S, Caglayan H and Ozbay E 2014 Coupling enhancement of split ring resonators on graphene Carbon 80 351-5

[48] Alici K B, Turhan A B, Soukoulis C M and Ozbay E 2011 Optically thin composite resonant absorber at the near-infrared band: a polarization independent and spectrally broadband configuration $O p t$. Express 19 14260-7

[49] Huang Y, Liu L, Pu M, Li X, Maa X and Luo X 2019 A refractory metamaterial absorber for ultra-broadband omnidirectional and polarization-independent absorption in the UV-NIR spectrum Nanoscale 10 8298-303

[50] Luo X 2020 Extraordinary young's interferences and super-diffraction laser lithography Handbook of Laser Micro-and Nano-Engineering ed K Sugioka (Berlin: Springer) pp 1-40

[51] Palik E and Ghosh G 1999 Handbook of Optical Constants of Solids II (New York: Academic)

[52] Johnson P B and Christy R W 2013 Optical constants of the noble metals Phys. Rev. B 15114003

[53] Caglayan H, Hong S H, Edwards B, Kagan C R and Engheta N 2013 Near-infrared metatronic nanocircuits by design Phys. Rev. Lett 111073904

[54] Hajian H, Ghobadi A, Butun B and Ozbay E 2017 Nearly perfect resonant absorption and coherent thermal emission by hBN-based photonic crystals Opt. Express 2531970 